\title{
Diabetes patient at risk score - a novel system for triaging appropriate referrals of inpatients with diabetes to the diabetes team 䇺
}

\author{
Authors: Rajesh Rajendran, ${ }^{\mathrm{A}}$ Rachael-Marie Round, ${ }^{\mathrm{B}}$ Christopher Kerry, ${ }^{\mathrm{C}}$ Sarah Barker ${ }^{\mathrm{D}}$ and Gerry Rayman ${ }^{\mathrm{E}}$
}

\begin{abstract}
The acceptability, uptake and effectiveness of a new referral tool - the diabetes patient at risk (DPAR) score - were evaluated and the timeliness of review of referred inpatients by the diabetes team was measured. For this, a snapshot survey of ward healthcare professionals (HCPs) and a review of all DPAR referrals to the diabetes team between 1 September 2013 and 31 January 2014 were undertaken. All referrals in November 2013 were audited for timeliness of review. 77\% of HCPs agreed/strongly agreed that the tool improved access to the diabetes team. $76 \%$ of referrals were from nurses. $80 \%$ of who should have been referred were referred; the remaining had already been reviewed by the diabetes team and therefore did not require referral. Only $11 \%$ of referrals were inappropriate. All DPAR referrals were reviewed within the stipulated time period in November 2013. Overall, the DPAR system was well accepted, successfully identified appropriate referrals and facilitated referrals in a timely manner to the diabetes team.
\end{abstract}

KEYWORDS: Inpatients, diabetes, Think Glucose, MEWS, inpatient audit

\section{Introduction}

The National Diabetes Inpatient Audit (NaDIA), the world's largest snapshot audit of inpatient diabetes care across England and Wales, revealed that only $8.9 \%$ of inpatients with diabetes were under the care of a diabetes consultant; the remainder were admitted for non-diabetes related conditions, eg hip fracture, pneumonia and myocardial infarction, under the care of non-diabetes specialty teams. ${ }^{1}$ It is well recognised that nondiabetes specialty teams may not have the expertise to maintain euglycaemia in all those under their care, for example those on complex insulin regimens, those with impaired renal function and those whose control has become destabilised because of the stress of illness and other reasons, such as altered hospital meal times and their composition. It is therefore important

\footnotetext{
Authors: A clinical research fellow, Ipswich Hospital, Ipswich, UK; ${ }^{B}$ diabetes inpatient specialist nurse, Ipswich Hospital, Ipswich, UK; ${ }^{C}$ diabetes inpatient specialist nurse, Ipswich Hospital, Ipswich, UK;

D diabetes inpatient specialist nurse, Ipswich Hospital, Ipswich, UK;

${ }^{E}$ consultant physician, Ipswich Hospital, Ipswich, UK
}

that non-diabetes specialist teams have appropriate and timely access to the expertise of inpatient diabetes teams for glycaemic advice and to the multidisciplinary diabetes foot team for active foot problems. An inpatient diabetes team should comprise of at least a consultant diabetologist, a podiatrist and a diabetes inpatient specialist nurse (DISN) devoting at least 4, 6 and 20 hours/week/100 beds respectively to inpatient diabetes care specifically. Ideally this team would also deliver weekend working which would therefore require a $50 \%$ increase in these hours. However, $31.7 \%$ of hospitals in the UK do not even have a dedicated DISN, and in those with DISNs the average time devoted to inpatients is only 7.6 hours/week/100 beds. ${ }^{1}$

The 'Think Glucose' programme was launched in 2009 by the NHS Institute for Innovation and Improvement to improve management of diabetes in inpatients. ${ }^{2}$ It included a traffic light referral system to help non-diabetes specialty teams identify those needing referral to the inpatient diabetes team. ${ }^{2}$ Inpatients with diabetes with certain specified problems or comorbidities who should always be referred were listed in the red group, those who may need referral in the amber group, and those who seldom need referral in the green group. After two years' experience of this system, a number of deficiencies were recognised which may explain the modest uptake and success in achieving appropriate and timely referrals to the inpatient diabetes team. The most significant of these was its failure to specify urgency. Thus, for example, inpatients with diabetic ketoacidosis (DKA) who require urgent review are in the same red category as inpatients who request a referral, those with newly diagnosed type-2 diabetes and those with diet-treated diabetes who are simply vomiting. Furthermore, the system does not account for inpatients with multiple problems. Thus, inpatients with several 'amber' problems/comorbidities remain amber even though, because of their complexity, are likely to need early review. To address these limitations, a simple referral tool was devised in this hospital, based on a numerical scoring system, in which each individual problem/complication is given a number, the sum of which indicates the urgency and priority for referral. Diabetic emergencies such as DKA, hyperosmolar hyperglycaemic state (HHS) and an acute red/hot/swollen foot are given the highest score which triggers immediate review if referred before $5 \mathrm{pm}$. Whereas for example, those due for early discharge but unable to self-manage and those who have had a severe hypoglycaemic episode would be less urgent, but 
Table 1. DPAR score (referral tool for all healthcare professionals to the diabetes team). Must be completed on admission and whenever concerned about diabetes care.

\begin{tabular}{|c|c|c|c|}
\hline Score & Glucose & Discharge assessment & Foot \\
\hline \multirow{2}{*}{$\begin{array}{l}8 \text { points } \\
\text { each }\end{array}$} & > DKA/HHS (HONK) & & $>$ New foot ulcer \\
\hline & > Newly disgnosed type-1 diabetes & & $>$ Acute red/hot/swollen foot \\
\hline \multirow{4}{*}{$\begin{array}{l}3 \text { points } \\
\text { each }\end{array}$} & $>$ New to s/c insulin therapy & > Unable to self-manage - expected & \\
\hline & $>$ Severe hypoglycaemia $\left(\mathrm{CBG}^{\mathrm{a}}<3 \mathrm{mmol} / \mathrm{l}\right)$ & discharge within 72 hours & \\
\hline & $>$ Recurrent hypoglycaemia $\left(\mathrm{CBG}^{\mathrm{a}}<4 \mathrm{mmol} / \mathrm{l}\right)$ & & \\
\hline & > Persistent raised $\mathrm{CBG}^{*} \geq 15 \mathrm{mmol} / \mathrm{l}$ & & \\
\hline \multirow{2}{*}{$\begin{array}{l}2 \text { points } \\
\text { each }\end{array}$} & > Insulin pump user & > Patient requests referral & $>$ Existing foot ulcer ${ }^{c}$ \\
\hline & $>$ On VRIII ${ }^{\mathrm{b}} \geq 48$ hours & $>$ Educational need & \\
\hline \multirow{2}{*}{$\begin{array}{l}1 \text { point } \\
\text { each }\end{array}$} & $>$ Commenced on steroids & & \\
\hline & > Commenced on parenteral/enteral feed & & \\
\hline
\end{tabular}

would be given a score triggering a review within 24 hours. All referrals were aimed to be reviewed within 24 hours as there was a seven-day inpatient diabetes team service. The exception was for foot reviews in which this was stipulated to be within one working day, as the foot team were not available over the weekends. When prioritising the referrals for review, the higher the cumulative score, the earlier the review by the inpatient diabetes team within the 24-hour period. This referral tool was termed the 'diabetes patient at risk' (DPAR) score (Table 1). Of note the 'Think Glucose' programme recommends referral whereas the DPAR score stipulates referral as well as urgency.

The DPAR referral tool is included in the diabetes care pathway booklet kept at the foot of the patient's bed. In addition to the DPAR tool, the booklet consists of a checklist designed to ensure that hypoglycaemia and DKA are recognised/avoided during admission, an assessment form to evaluate the patient's competence at self-administration of insulin and blood glucose testing, a foot protection programme, a subcutaneous insulin prescription chart, a capillary glucose monitoring record, an intravenous insulin infusion prescription and administration chart, a safe diabetes discharge checklist and guidance on managing hyperglycaemia and hypoglycaemia. The DPAR referral tool was introduced across all wards towards the end of August 2013 and is used in every inpatient with diabetes. If the score indicates a referral, this is made via an electronic DPAR referral system (Fig 1) which also permits additional details to be included, and logs time from referral to review, as well as details of the action of the inpatient diabetes team. In addition to the electronic referral, immediate verbal referral to the diabetes team via dedicated diabetes 'bleep' is required for all diabetic emergencies such as DKA, HHS and an acute red/hot/ swollen foot within working hours. Any healthcare professional (HCP) on the ward can refer using the DPAR system, and all referrals are streamlined to a single member of the team who re-allocates it to one or more appropriate members. For example, an inpatient with persistent hyperglycaemia and an existing foot ulcer would be reviewed by both a DISN and a podiatrist. The aims of this study were to evaluate the acceptability and uptake of the DPAR referral system by various groups of HCPs, its effectiveness in triggering appropriate referrals and that it leads to timely review.

\section{Methods and materials}

To determine the acceptability of the new referral system among ward doctors and nurses, a one-day snapshot survey was performed in September 2013 and repeated in June 2014. To determine the uptake of the DPAR referral system by various HCP groups, all referrals between 1 September 2013 and 31 January 2014 were reviewed to categorise the referrer. To determine the effectiveness of the DPAR tool as a referral system, the inpatient diabetes team reviewed all inpatients with diabetes between 1 September 2013 and 31 January 2014, assessed their need for referral according to the DPAR score, whether they had been referred and whether the referrals were appropriate. The timeliness of review by the inpatient diabetes team for diabetic emergencies (ie those needing immediate review) were evaluated for the same period between 1 September 2013 and 31 January 2014. Timeliness of review for all DPAR referrals was evaluated for the month of November 2013 only.

\section{Results}

\section{Acceptability}

In September 2013, 94 of 95 HCP surveyed (23\% medical, 77\% nursing) gave feedback on the DPAR system. Responses to the question asking whether the DPAR system improved access to the inpatient diabetes team were extremely positive: strongly agree (13), agree (59), neither agree nor disagree (18), disagree (4) and strongly disagree (0). In June 2014, 105 of 105 surveyed (20\% medical, $80 \%$ nursing) gave similar feedback: strongly agree (22), agree (60), neither agree nor disagree (17), disagree (6) and strongly disagree (0). 


\section{The Ipswich Hospital WHS

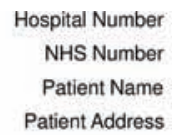

Postcode

DOB/Gender

Ward

Select One

DIABETES PATIENTS AT RISK (DPAR) SCORE

(Referral tool for all healthcare professionals to the Diabetes team)

Immediately refer all patients with a score $>=2$ electronically

If score is $>=8$ the Diabetes consultant (bp 140) should be bleeped in addition to electronic referral

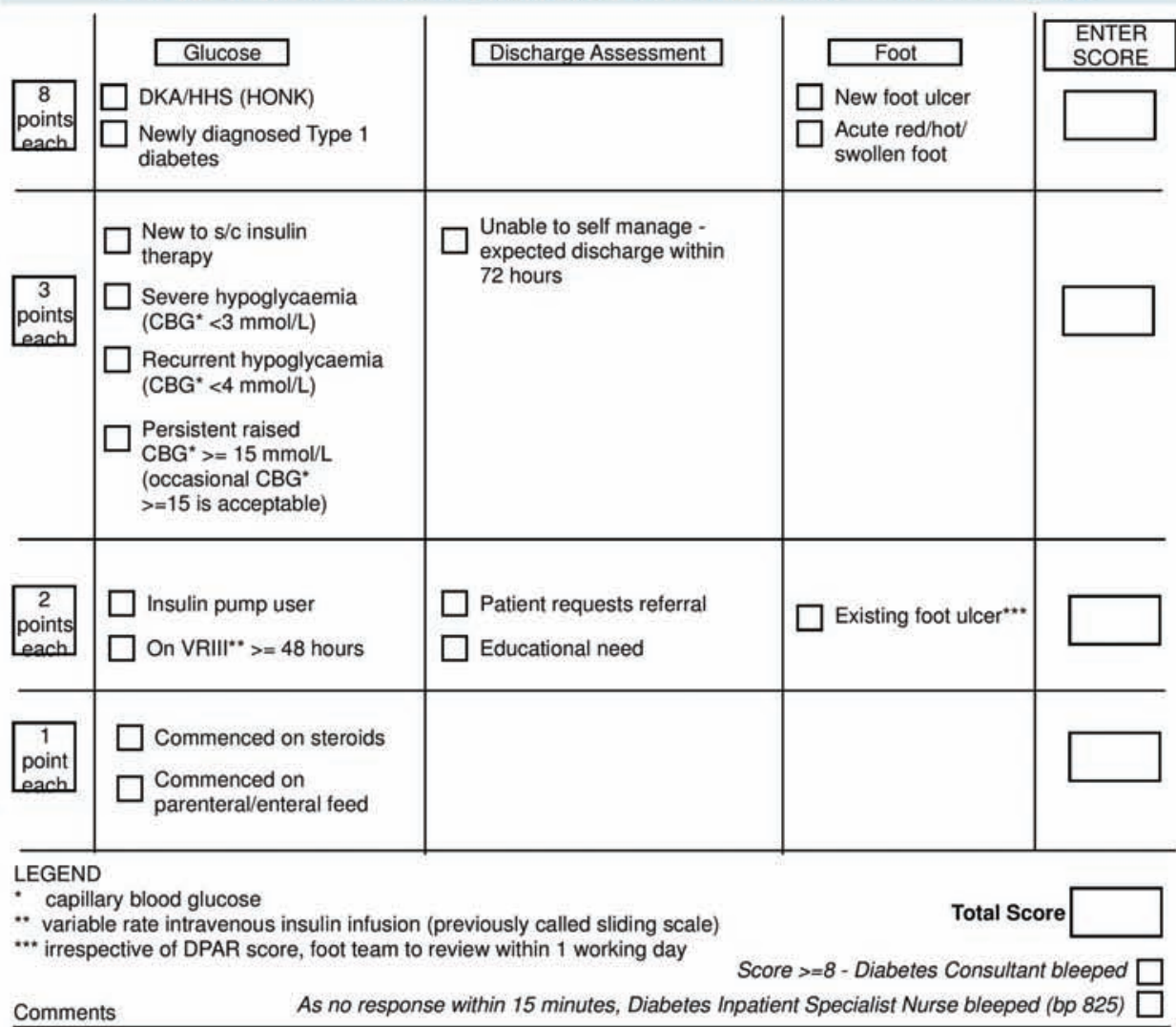

Comments

Form Completion Date $\quad$ Form Completed By

DRAFT 8
Fig 1. DPAR electronic referral form.

\section{Uptake}

Between 1 September 2013 and 31 January 2014, there were 471 DPAR referrals of which 395 were appropriate, 53 were inappropriate and 23 were duplicate referrals. Of the 395 appropriate referrals, 299 (76\%) were from nurses, 95 from doctors and 1 from an allied HCP. The majority of inappropriate referrals $(>90 \%)$ related to foot issues that did not require review by the diabetes team, for example inpatients requiring trimming of the nails and inpatients with newly diagnosed insensate feet but no ulceration who simply required heel pressure ulcer prevention. All such referrals were from nursing staff.

\section{Effectiveness}

Between 1 September 2013 and 31 January 2014, of all inpatients with diabetes, 494 were considered by the inpatient diabetes team to require a diabetes review, of whom $395(80 \%)$ had been referred via the DPAR system and all were reviewed 
Table 2. Data from the NaDIA for the hospital studied. ${ }^{1}$

\section{Parameter}

Referral system in use

Inpatients included in the week (September) of the national audit, $\mathrm{n}$

Inpatients requiring inpatient diabetes team review, $\mathrm{n}$ (\%)

Inpatients seen who required review by a member of the inpatient diabetes team, $\mathrm{n}(\%)$

Average good diabetes days per patient per week, $n$

Medication errors, \% charts audited

Prescription errors, \% charts audited

Management errors, \% charts audited

Insulin errors, \% charts audited

Severe hypoglycaemia (capillary blood glucose $<3 \mathrm{mmol} / \mathrm{l}$ ), \% inpatients audited

\section{NaDIA 2012}

Think Glucose traffic light referral system

86

$44(51.2)$

$28(63.6)$

$3.8 / 7$

56.9

29.3

43.1

31

$15.4 \%$
NaDIA 2013

DPAR scoring and referral system

96

54 (56.2)

$50^{a}(92.6)$

$5.8 / 7$

21.1

12.7

11.3

7

$9.7 \%$

${ }^{a}$ Remaining 4 inpatients were awaiting review at the time of the audit and were seen by the inpatient diabetes team later in the day. DPAR = diabetes patient at risk; NaDIA = National Diabetes Inpatient Audit.

subsequently by the inpatient diabetes team. The remaining $99(20 \%)$ had already been seen by the inpatient diabetes team during their daily ward visits and therefore did not require separate referral. It was therefore not possible to evaluate whether these 99 inpatients would have been referred or not, if they had not been previously reviewed by the inpatient diabetes team.

\section{Timeliness of review following referral}

\section{Diabetic emergencies}

All 29 patients with DKA, HHS and all newly diagnosed type-1 patients admitted during the audit period were referred via the DPAR process; 23 referred before $5 \mathrm{pm}$ were seen within the hour of referral; the 6 referred after $5 \mathrm{pm}$ were seen the next morning.

\section{All DPAR referrals}

In November 2013, out of 113 DPAR referrals there were 34 that required review by the foot team; 30 were seen within 24 hours and 4 within 48 hours but not within 24 working hours; all within the target time period for review by the foot team. The remaining 79 referrals for metabolic and educational issues, such as hypoglycaemia and hyperglycaemia, were also reviewed within the target time frame of 24 hours and in the order of priority according to the score on the DPAR referral system.

\section{Discussion}

Further to recommendations from the Department of Health, the National Institute for Health and Care Excellence and the Royal College of Physicians, the National Early Warning Score (NEWS) is universally used across all NHS Trusts to efficiently identify and react to patients with acute illness. ${ }^{3-5}$ Two important reasons for its universal espousal are its simplicity (including ease of calculation of total score) and ability to allow the HCP to prioritise review based on the score in a busy hospital environment. The same principles were used to devise the DPAR triaging system. This may be the reason for the good uptake among various HCPs and their positive feedback from the surveys.

It was noted that when the 'Think Glucose' criteria suggested referral, nurses usually consulted medical staff before the referral was made. This not only delayed referral but nondiabetes medical staff often overruled the decision. The DPAR referral system, like the NEWS overcomes this by empowering nurses to refer directly and with a measured degree of timeliness. Furthermore, like the NEWS, the score can be updated. Direct referral from nurses to the inpatient diabetes teams are important as they are more closely involved in bedside care and are more likely to be quickly aware of change in status. Its empowerment of nurses is evident by the higher percentage of referrals from nurses compared with doctors, whereas traditionally when the Think Glucose system was in use, the referrals were usually only from the doctors. $11 \%$ of DPAR referrals were inappropriate and were from nurses. Direct feedback was given to the HCP who completed these inappropriate referrals and as a result inappropriate referral rates are now no more than 1-2 per month.

One of the limitations in this study was not to have made a direct comparison of the DPAR referral system with the Think Glucose system. Nevertheless, data were available from the nurses' log of referrals, which showed that in the same time period in the previous year (between 1 Septemeber 2012 and 31 January 2013) when the Think Glucose referral system was being used, there were only 194 referrals. Unfortunately, the number of inpatients who required review was not available nor was it possible to obtain complete clinical details to judge the appropriateness of the referrals. Assuming that the number of inpatients with diabetes who would have needed a review, would be very similar to that in the subsequent year, the 194 referrals when the Think Glucose system was in operation, suggests that only $39 \%$ of those who required referral were referred. Recently, Ryder et al showed that incorporating the 'Think Glucose' system in their hospital electronic investigation ordering system increased reviews by the inpatient diabetes team from 83 to 452 per month. ${ }^{6}$ However, the number of referrals made, the number that were inappropriate, their 
method of prioritisation of reviews and whether they were seen within specified time frames were not reported.

There are limitations to our scoring system. Other diabetes teams may score clinical situations differently; nevertheless, the ability to weigh the urgency is of significant clinical value particularly for diabetic emergencies, where linking this to a speedy review process resulted in nearly $80 \%$ of these being seen by the diabetes team within one hour of referral when referred within working hours. The apparent success of the referral system may be due to other issues such as the drive to increase awareness of inpatient diabetes care, and as such, the system needs piloting elsewhere. Furthermore, the impact on clinical outcomes such as glucometrics, medication errors and morbidity will need to be demonstrated. In this respect it should be noted that, at present, the clinical outcomes of the widely adopted Think Glucose system have not been evaluated. To date, many hospitals have been using their yearly NaDIA data to benchmark themselves as well as to compare themselves against other hospitals and against the whole country. ${ }^{1}$ Given its limitation of being a snapshot audit, it may still be of interest that, following introduction of the DPAR system there were improvements in the NaDIA results for the hospital studied (Table 2). The NaDIA 2012 and 2013 audits for this hospital showed a significant increase in the percentage of those needing review that were actually reviewed; from $63.6 \%$ (when the Think Glucose referral system was in place) to $92.6 \%$ (following introduction of the DPAR referral system). By contrast, nationally there was no significant change (58.5\% in 2012 and $62.5 \%$ in 2013). ${ }^{1}$ The improvement in the hospital's NaDIA results is in keeping with the observation of a more than twofold increase in referrals after introduction of the DPAR scoring system.

In conclusion, this study demonstrates that the DPAR system is well accepted by ward staff and successfully identifies appropriate referrals of inpatients with diabetes, enhancing their timely review by the inpatient diabetes team. Successful implementation in other NHS Trusts will determine its true value.

\section{Funding}

The Ipswich Diabetes Inpatient Care and Education (DICE) project has received a grant from Sanofi, who have not been involved in the design and origin of this study, the inpatient diabetes care pathway or the analysis, the interpretation and reporting of the data and in preparation of this manuscript.

\section{Acknowledgements}

We thank all members of the DICE team (Gerry Rayman (lead), Rajesh Rajendran, Damian Morris, Craig Parkinson, Duncan Fowler, Anne Scott, Sue Mitchell, Christopher Kerry, Rachael-Marie Round, Sarah Barker, Richard Driver, Maggie Rudduck and Karl Kroger) for their contribution to development and implementation of the DPAR referral system as part of the Ipswich DICE project.

\section{References}

1 Health and Social Care Information. National diabetes inpatient audit. Leeds: HSCIC, 2014. Available online at www.hscic.gov.uk/ diabetesinpatientaudit [Accessed 17 March 2015].

2 NHS Institute for Innovation and Improvement. Think glucose: inpatient care for people with diabetes. London: NHS, 2009. Available online at www.institute.nhs.uk/quality_and_value/think_glucose/ welcome_to_the_website_for_thinkglucose.html [Accessed 17 March 2015].

3 Expert Group. Comprehensive critical care: a review of adult critical care services. London: DH, 2000. Available online at http:// webarchive.nationalarchives.gov.uk/20130107105354/http://www. dh.gov.uk/prod_consum_dh/groups/dh_digitalassets/@dh/@en/ documents/digitalasset/dh_4082872.pdf [Accessed 17 March 2015].

4 National Institute for Health and Care Excellence. Acutely ill patients in hospital: recognition of and response to acute illness in adults in hospital (CG50). London: NICE, 2007. Available online at www.nice.org. uk/guidance/cg50/resources/guidance-acutely-ill-patients-inhospital-pdf [Accessed 17 March 2015].

5 Royal College of Physicians. National Early Warning Score (NEWS): Standardising the assessment of acute-illness severity in the NHS. Report of a working party. London: RCP, 2012.

6 Ryder B, Burbridge W, Braycotton L et al. Inpatient diabetes: do-ityourself electronic referral system to support and enhance the Think Glucose project. Prac Diabetes 2014;31:194-6.

Address for correspondence: Dr G Rayman, Diabetes Centre, The Ipswich Hospital NHS Trust, Heath Road, Ipswich IP4 5PD, UK.

Email: gerry.rayman@ipswichhospital.nhs.uk 